Ingeniare - Revista Chilena de Ingeniería, vol. $14 \mathrm{~N}^{\mathrm{o}} 2,2006, \mathrm{pp} .153-158$

\title{
USO DE CADENAS DE MARKOV PARA LA PREDICCIÓN DE LA DINÁMICA DEL COMPORTAMIENTO DE PACIENTES EN UNA UNIDAD DE CUIDADO INTENSIVO CARDIOLÓGICA
}

\section{USE OF MARKOV CHAINS IN PREDICTION OF THE DYNAMICS OF PATIENT BEHAVIOR IN A CARDIOLOGIC INTENSIVE CARE UNIT}

\author{
Víctor Albornoz $^{1}$ Mónica Hinrichsen $^{2}$ Patricia Miranda ${ }^{2} \quad$ Pedro Peña $^{1}$ \\ Recibido 29 de junio de 2005, aceptado 6 de junio de 2006 \\ Received: June 29, 2005 Accepted: June 6, 2006
}

\begin{abstract}
RESUMEN
En este trabajo presentamos un modelo probabilístico que contribuye al estudio de la dinámica en el comportamiento y permanencia de pacientes en una unidad de cuidados intensivos cardiológica. El modelo utilizado corresponde a una Cadena de Markov en tiempo discreto, que mediante la definición de determinados niveles de gravedad de un paciente (estados) y la obtención de las correspondientes probabilidades de transición entre un nivel de gravedad y otro, permite predecir los tiempos de permanencia. Los diferentes estados empleados se basan en la construcción de un nuevo score creado para este propósito. Se muestran los detalles de la metodología adoptada y los principales resultados alcanzados en la aplicación del modelo empleado.
\end{abstract}

Palabras clave: Cadenas de Markov, modelo probabilístico, unidad de cuidado intensivo, permanencia de pacientes, score.

\section{ABSTRACT}

In this paper we present a probabilistic model that contributes to the study of dynamics in the behavior and permanence of patients in a cardiovascular intensive care unit. The model corresponds to a discrete Markov Chain, that allows to predict the time that a patient remains in the system through the time, by means of certain severity of illness states and the corresponding transition probabilities between those states. The different states are based on the construction of a new score created for this study. We summarize the details of the adopted methodology and the main results reached in the application of the model.

Keywords: Markov chains, probabilistic model, intensive care unit, length of hospital stay, score.

\section{INTRODUCCIÓN}

En este trabajo empleamos un modelo de naturaleza probabilística para predecir la duración promedio en la permanencia de un paciente en la Unidad de Cuidados Intensivos Cardiológica del Hospital Dr. Luis Calvo Mackenna (UCIC). Del mismo modo, el modelo empleado permite estudiar la evolución de un paciente a través de los diferentes niveles de gravedad (estados) contemplados en este estudio, cuyo conocimiento se espera contribuya posteriormente a la gestión de dicha unidad de salud.
El modelo empleado corresponde más específicamente a una Cadena de Markov en tiempo discreto, comúnmente utilizada en el ámbito de la Investigación de Operaciones para describir y predecir el comportamiento de ciertos sistemas bajo condiciones de incertidumbre a través del tiempo. La utilización de estos modelos ha resultado adecuada para modelar dinámica de poblaciones, sistemas de espera, control de inventarios, mantenimiento y reemplazo de equipos y en apoyo a la toma de decisiones en administración, ingeniería y medicina $[1,2$ y 3$]$.

\footnotetext{
1 Departamento de Industrias. Universidad Técnica Federico Santa María. Av. Santa María 6400. Santiago. Chile. E-mail: victor.albornoz@usm.cl

2 Hospital Dr. Luis Calvo Mackenna. Servicio de Cardiología. Av. Antonio Varas 360. Santiago. Chile.
} 
Entre las aplicaciones de procesos markovianos en el ámbito de la salud, podemos citar, por ejemplo, los trabajos de Aikawa et al. [4], que mide el efecto económico que provoca el uso de una determinada droga en la permanencia de un paciente con cierto síndrome en una unidad de cuidado intensivo; Bauerle et al. [5], que corresponde a un estudio predictivo y comparativo -por sexo y edades- en las muertes por sepsis en unidades de cuidado intensivo; Collart y Haurie [6], que emplean un proceso markoviano para tomar decisiones óptimas en la admisión de pacientes en una unidad urológica; Launois et al. [7], que estudia desde una perspectiva económica la conveniencia de establecer unidades específicas para pacientes con infartos cuya evolución se describe a través de una Cadena de Markov; Patten [8], que usa cadenas de Markov para estudiar la evolución de pacientes con depresión, y Shmueli et al. [ 9], que modelan la llegada y tiempos de permanencia de pacientes a través de un proceso markoviano en tiempo continuo y contrastan tres políticas de admisión de pacientes para obtener una política eficiente que incremente el número de sobrevivientes.

La predicción de la duración en la permanencia de los pacientes en una unidad de cuidado intensivo permite planificar adecuadamente la atención de pacientes y ha sido materia de estudio en numerosos trabajos, que comprenden la utilización de índices de gravedad, métodos estadísticos y modelos probabilísticos. Los índices generales de gravedad de un paciente como APACHE (Acute Physiology and Chronic Health Evaluation), SAPS (Simplified Acute Physiology Store), MPM (Mortality Probability Models) y Parsonnet Score [10, 11, 12 y 13] describen el comportamiento del paciente en lo inmediato, en base a su estado de ingreso o una vez intervenido quirúrgicamente, y son empleados para predecir el riesgo y mortalidad de pacientes con enfermedades cardiológicas, pero también para estudiar su permanencia en unidades de cuidado intensivo basada en esa misma información [14, 15]. Por otra parte, una gran cantidad de trabajos predice la estadía de pacientes a través de métodos estadísticos lineales [16, 17, 18 y 19]. La principal desventaja de estos últimos es que no incorporan las complejas interacciones existentes entre las numerosas variables que determinan tal permanencia, aspecto que corrigen modelos predictivos no-lineales como las redes neuronales artificiales [20, 21 y 22]. Por último, entre los modelos probabilísticos destaca igualmente el empleo de cadenas de Markov para abordar este problema [23, 24], artículos estos últimos que hemos tomado como base para el presente trabajo.

En este artículo, en particular, formulamos y utilizamos una Cadena de Markov en tiempo discreto para el estudio del comportamiento de pacientes en la UCIC. En esta cadena hemos definido un número finito de estados que representan el grado de enfermedad de los pacientes. Los estados están asociados a un determinado nivel de riesgo y permiten describir la evolución de los pacientes, en base a un score asignado por su diagnóstico, tipo de intervención quirúrgica al que ha sido sometido el paciente y las complicaciones postoperatorias. En lo que sigue, el trabajo se organiza de la siguiente manera. La próxima sección describe brevemente la metodología empleada. A continuación, describimos algunos detalles de la aplicación y los resultados alcanzados. Finalmente, enumeramos las principales conclusiones de este trabajo.

\section{MÉTODO}

Una Cadena de Markov corresponde a una clase específica de proceso estocástico en el ámbito de modelos probabilísticos $[25,26]$. En este trabajo $x_{t}$ denotará el estado de un paciente en el instante de tiempo futuro t. Esto define un proceso estocástico que corresponde a la secuencia $x_{0}, x_{1}, x_{2}, x_{3}, \ldots$, que representa su nivel de gravedad a través del tiempo, en la cual usualmente el valor de un $x_{t}$ depende de los valores previos en la secuencia. A medida que transcurre el tiempo, los cambios de estado tienen lugar en términos probabilísticos y son representados a través de las denominadas probabilidades de transición entre estados, que en el caso de las transiciones en una etapa corresponde a la probabilidad de pasar de un estado a otro desde una etapa de tiempo $t$ a la siguiente $t+1$.

Las hipótesis que contempla este modelo markoviano en discusión son las siguientes: 1) supone un número finito de estados para describir el comportamiento dinámico de los pacientes; 2) supone conocida una distribución de probabilidades al inicio del horizonte de estudio $(\mathrm{t}=0)$, que refleje ya sea a qué estado de los previamente definidos pertenece un paciente de la UCIC, o bien, los porcentajes de pacientes en cada estado en la UCIC; 3) supone que la transición de un estado actual a otro en el futuro depende solamente del estado actual (propiedad markoviana), y 4) que la probabilidad de esta transición sea independiente de la etapa de tiempo considerada (propiedad estacionaria), esto es, que no cambie en el tiempo de estudio del sistema. Naturalmente, la validez del modelo y las respuestas entregadas dependen esencialmente del cumplimiento de las propiedades markoviana y estacionaria, por ende, resulta necesario un análisis para establecer cuán adecuadas son en cada caso particular.

Para definir los estados de la cadena se empleará un determinado índice o score, asociado a un nivel de 
riesgo y gravedad de un paciente en la UCIC. Para determinar este score se toman en cuenta seis factores: edad, condición inicial, diagnóstico de gravedad, cirugía previa, intervención quirúrgica y complicaciones postoperatorias. Los cuatro primeros tienen relación con el estado del paciente al ingreso, en tanto los dos últimos dan cuenta de la evolución del paciente en su permanencia en la UCIC. A cada uno de estos factores se asigna un score dentro de un cierto rango. El rango no es el mismo para cada factor, pues permite establecer diferencias relativas entre los distintos aspectos considerados. Naturalmente, el score asignado en cada caso queda determinado, a su vez, por diferentes niveles de riesgo o gravedad dentro de cada factor. Así, por ejemplo, para un factor como la intervención quirúrgica, un cierre de ductus o una reparación de coartación tienen un score 4; en tanto una operación Norwood, una reparación de tronco arterioso 1 ó 2; un doble switch o un Jatene con reparación de interrupción de cayado aórtico tienen un score 36. A su vez, en un factor epidemiológico como la edad el rango va desde un score 2 para mayores de dos años a un score 8 para niños menores de un mes.

La importancia relativa entre los distintos factores se consigue definiendo un score máximo que cambia con la importancia del factor considerado, el score máximo para la edad es 8 , para una cirugía previa 8 , para la condición inicial es 12, para las complicaciones postoperatorias 16, para el diagnóstico 24 y para el tipo de intervención quirúrgica 36. La figura 1 muestra los rangos de variación del score para los distintos aspectos que determinan el estado de un paciente, gráfica que permite apreciar tanto la importancia relativa entre ellos así como el hecho de que los factores iniciales y aquellos que determinan su evolución tienen, a su vez, la misma importancia relativa.

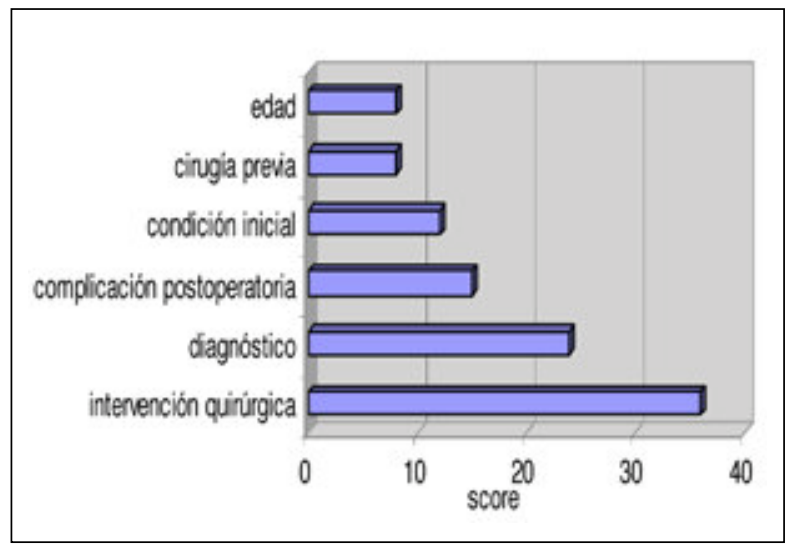

Figura 1. Rangos de variación del score para cada factor.
A partir de la suma de los scores para cada aspecto particular, se obtiene el score de un paciente para cada etapa de tiempo t en que permanece en la UCIC. Este score determina finalmente la clasificación de la gravedad de un paciente, etapa por etapa, en cualquiera de los siguientes estados: Estado A (riesgo bajo, score $\leq 25$ ), Estado $\mathbf{B}$ (riesgo medio, $26 \leq$ score $\leq 41$ ), Estado $\mathbf{C}$ (riesgo alto, $42 \leq$ score $\leq 57$ ) y Estado $\mathbf{D}$ (riesgo grave, score $\geq 58$ ). Adicionalmente, a los estados anteriores se agrega un Estado E, para indicar que un paciente ya abandonó la UCIC en alguna etapa de tiempo.

De esta manera, a partir de datos históricos con toda la evolución de un paciente particular, desde el momento en que ingresa a la UCIC hasta que la abandona, digamos al cabo de $\mathrm{T}$ etapas de tiempo (para cada ingreso a la UCIC por separado), podemos resumir la dinámica de su comportamiento a través de una secuencia conocida (trayectoria) como ser: $\mathrm{x}_{0}=\mathbf{C}, \mathrm{x}_{1}=\mathbf{C}, \mathrm{x}_{2}=\mathbf{D}, \mathrm{x}_{3}=\mathbf{B}$, $\mathrm{x}_{4}=\mathbf{B}, \ldots, \mathrm{x}_{\mathrm{T}-1}=\mathbf{A}, \mathrm{x}_{\mathrm{T}}=\mathbf{E}$.

Una vez construido el conjunto de secuencias para todos los ingresos a la UCIC registrados en una base de datos histórica de cierto periodo, se procede al cálculo de las probabilidades de transición entre estados. En lo que sigue, denotamos por $p_{i j}$ la probabilidad de transición (en una etapa) desde el estado $i$ al estado $j$, con $i=\mathbf{A}, \mathbf{B}, \mathbf{C}, \mathbf{D}$ y $\mathbf{j}=\mathbf{A}, \mathbf{B}, \mathbf{C}, \mathbf{D}, \mathbf{E}$. La probabilidad $p_{i j}$ resulta de calcular el cuociente entre la cantidad total de transiciones desde el estado $i$ al estado $j$ y el total de dichas transiciones que simplemente se inician en el estado $i$, considerando todas aquellas transiciones (en una etapa) que tengan lugar en cualquier etapa del conjunto de secuencias. Adicionalmente, definimos las siguientes probabilidades de transición para el estado $\mathbf{E}: p_{E A}=0, p_{E B}=0, p_{E C}=0, p_{E D}=0$ y $p_{E E}=1$. Como es usual, las respectivas probabilidades se resumen en la matriz de probabilidades de transición (en una etapa) $\mathrm{P}=\left(p_{i j}\right)_{\mathrm{i}, \mathrm{j}=\mathrm{A}, \mathrm{B}, \mathrm{C}, \mathrm{D}, \mathrm{E}}$

Por último, cabe destacar de la teoría de Cadenas de Markov que la matriz $\mathrm{P}^{\mathrm{n}}$ (P elevada a la n-ésima potencia) provee, a su vez, las probabilidades de transición al cabo de n-etapas, probabilidades que denotamos por $p_{i j}{ }^{(n)}$, necesarias para conocer en términos probabilísticos la dinámica de la permanencia de un paciente que ingresa a la UCIC en uno cualquiera de los estados considerados, como se verá en la siguiente sección.

\section{RESULTADOS}

En este artículo se considera una muestra de 64 ingresos diferentes, cuyos datos fueron registrados en la UCIC durante un periodo de aproximadamente 14 meses y 
con pacientes cuyas estadías variaron entre 1 y 32 días y que sirvió como base para el trabajo de Peña [27] en el estudio y aplicación de estas metodologías.

Para simplificar los cálculos realizados, cada etapa de la Cadena de Markov corresponde a un periodo de dos días y los datos considerados abarcan pacientes que estuvieron entre una y 16 etapas. Así entonces, para cada uno de los 64 ingresos se calculó el score de cada paciente en cada etapa de su permanencia, lo que determinó, a su vez, la secuencia de estados en el sistema. De las 64 secuencias se obtuvo la siguiente matriz de probabilidades de transición (en una etapa), cuyos elementos $p_{i j}$ corresponden a la probabilidad de pasar de un estado $i$ a un estado $j$ al cabo de dos días:

$\mathrm{P}=\left[\begin{array}{ccccc}103 / 169 & 8 / 169 & 1 / 169 & 0 & 57 / 169 \\ 46 / 194 & 114 / 194 & 22 / 194 & 10 / 194 & 2 / 194 \\ 11 / 50 & 18 / 50 & 18 / 50 & 2 / 50 & 1 / 50 \\ 0 & 6 / 14 & 2 / 14 & 2 / 14 & 4 / 14 \\ 0 & 0 & 0 & 0 & 1\end{array}\right]$

Notar que la cadena resultante tiene dos clases de estados: una clase de estados transientes, formada por los estados A,B,C y $\mathbf{D}$, y una clase de estados recurrentes, formada por el estado absorbente E. Además, la cadena posee una distribución estacionaria que indica que con probabilidad 1 el paciente hará abandono de la UCIC en el largo plazo.

En lo que sigue, hacemos la predicción de la permanencia en la UCIC de un paciente que ingresa en un estado cualquiera. Para ello, definimos primeramente la variable aleatoria (v.a.) discreta $X_{\mathrm{ij}}(\mathrm{n})$, que toma el valor 1 , si un paciente que ingresa en el estado i está en el estado $\mathrm{j}$ al cabo de $\mathrm{n}$ etapas y el valor 0 , en caso contrario. Claramente, se verifica que $\mathrm{E}\left(\mathrm{X}_{\mathrm{ij}}(\mathrm{n})\right)=p_{i j}{ }^{(n)}$, esto es, el valor esperado de dicha v.a. es simplemente la probabilidad de transición del estado $i$ al estado $j$ al cabo de $n$ etapas, probabilidad que puede ser obtenida a partir de la componente $(\mathrm{i}, \mathrm{j})$ de la matriz $\mathrm{P}^{\mathrm{n}}$.

Enseguida, definimos la v.a. $\mathrm{V}_{\mathrm{ij}}(\mathrm{n})=\sum_{\mathrm{m}=1, \mathrm{n}} \mathrm{X}_{\mathrm{ij}}(\mathrm{m})$ como el número de veces que el paciente, que ingresó en el estado i, estuvo en el estado $\mathrm{j}$ durante $\mathrm{n}$ etapas. Dado lo anterior, $\mathrm{E}\left(\mathrm{V}_{\mathrm{ij}}(\mathrm{n})\right)=\mathrm{E}\left(\sum_{\mathrm{m}=1, \mathrm{n}} \mathrm{X}_{\mathrm{ij}}(\mathrm{m})\right)=\sum_{\mathrm{m}=1, \mathrm{n}} p_{i j}^{(m)}$ representa el número esperado de etapas que un paciente, que ingresó en el estado $i$, estuvo en el estado $j$, considerando un total de $\mathrm{n}$ etapas de transición.

Luego, para cada estado inicial $i$, definimos $\mathrm{V}_{\mathrm{i}}(\mathrm{n})=$
$\sum_{j=A, B, C, D} E\left(V_{i j}(n)\right)$ que representa la cantidad de etapas que un paciente estuvo en el sistema, dado que ingresó en el estado $i$. Para un valor suficientemente grande de $n$, que denotamos por $n=\infty, V_{i}(\infty)$ indica cuántas etapas (en promedio) estuvo un paciente cualquiera que ingresó en un estado inicial dado $i$. Para $n=16$ en el problema en estudio, los valores de $\mathrm{V}_{\mathrm{i}}(16)$ corresponden más precisamente a:

$$
\begin{aligned}
& \mathrm{V}_{\mathrm{A}}(16)=3.002 \text { etapas } \approx 6.0 \text { días, } \\
& \mathrm{V}_{\mathrm{B}}(16)=6.328 \text { etapas } \approx 12.7 \text { días, } \\
& \mathrm{V}_{\mathrm{C}}(16)=5.996 \text { etapas } \approx 12.0 \text { días, } \\
& \mathrm{V}_{\mathrm{D}}(16)=4.402 \text { etapas } \approx 9.0 \text { días. }
\end{aligned}
$$

Los valores de $\mathrm{V}_{\mathrm{i}}(\infty)$ permiten calcular la estadía promedio estimada de un paciente cualquiera (ep). En efecto, suponiendo conocida una distribución inicial $\mathrm{f}_{0}$, donde $\mathrm{f}_{\mathrm{i} 0}$ representa la probabilidad de que un paciente ingrese en el estado $i$, resulta entonces $e p=\sum_{\mathrm{j}=\mathrm{A}, \mathrm{B}, \mathrm{C}, \mathrm{D}} \mathrm{f}_{\mathrm{i} 0} \mathrm{~V}_{\mathrm{j}}(\infty)$. Usando los valores de $\mathrm{V}_{\mathrm{i}}(16)$, para aproximar los de $\mathrm{V}_{\mathrm{i}}(\infty)$, y la distribución inicial a la que da origen los datos de la muestra de 64 pacientes, se tiene un valor estimado de etapas en la estadía de 5.824 etapas (correspondiente a 11.6 días).

Notar que, al definir $\mathrm{v}_{\mathrm{j}}(\mathrm{n})=\sum_{\mathrm{i}=\mathrm{A}, \mathrm{B}, \mathrm{C}, \mathrm{D}} \mathrm{f}_{\mathrm{i} 0}\left(\sum_{\mathrm{m}=1, \mathrm{n}} p_{i j}^{\left({ }^{(m)}\right.}\right)$, que representa el total (esperado) de etapas que un paciente estuvo en el estado $\mathrm{j}$, independientemente de su estado inicial, considerando un total de $n$ etapas de transición, con un valor de $\mathrm{n}$ suficientemente grande, digamos $\mathrm{n}=\infty$, la estadía promedio equivale igualmente a $e p=\sum_{\mathrm{j}=\mathrm{A}, \mathrm{B}, \mathrm{C}, \mathrm{D}} \mathrm{v}_{\mathrm{j}}(\infty)$.

Con el propósito de validar la metodología adoptada se dividió la muestra inicial en dos grupos de 32 pacientes cada uno, elegidos al azar. A partir de los datos de uno de los grupos se hicieron los cálculos anteriores para obtener la estadía promedio estimada usando valores de $n=1,2, \ldots, 16$ etapas. Con los datos del otro grupo se construyeron los valores de la llamada estadía promedio observada para cada valor de n. Estos últimos resultan de calcular primeramente el número de veces que se visitó un determinado estado $\mathrm{j}$ en las $\mathrm{n}$ etapas de toda la muestra de este grupo (considerando las secuencias truncadas de hasta $n$ etapas) dividido por el tamaño de la muestra, cuya posterior suma tomando todos los valores posibles de $\mathrm{j}=\mathrm{A}, \mathrm{B}, \mathrm{C}$ y D arroja la estadía observada. La figura. 2 permite apreciar las diferencias entre la permanencia promedio observada y la estimada por el modelo, con los respectivos grupos de 32 pacientes. La diferencia arrojada para ambos valores no es estadísticamente significativa con $p=0.05$. 
Albornoz et al.: Uso de cadenas de Markov para la predicción de la dinámica del comportamiento de pacientes en una unidad de cuidado...

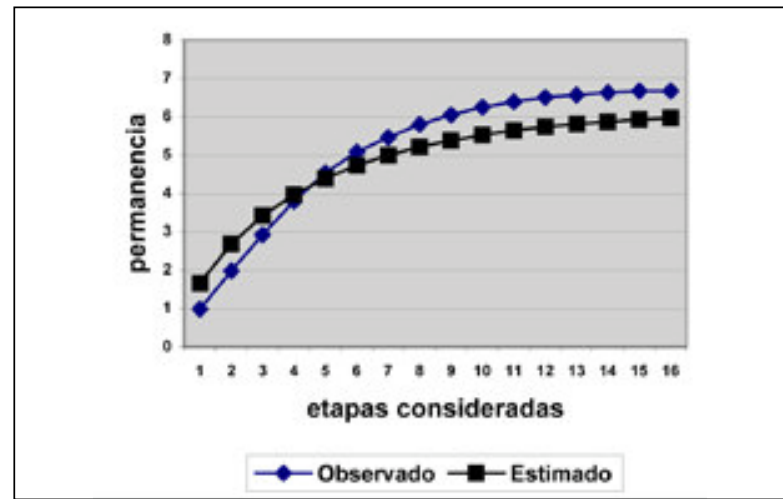

Figura 2. Diferencias entre lo observado y lo pronosticado por el modelo.

\section{CONCLUSIONES}

En el presente trabajo proponemos un modelo markoviano para predecir la permanencia de pacientes en una UCIC. La metodología adoptada permite enfrentar la incertidumbre presente en esta clase de problemas, describiendo la dinámica de la permanencia de los pacientes en términos probabilísticos.

El modelo empleado resulta satisfactorio para abordar el problema en estudio, tomando en cuenta su comportamiento y las diferencias obtenidas en comparación con la muestra de datos con la que se contrastó. De igual forma, la propuesta de un score y los diferentes estados considerados permiten reflejar adecuadamente las complicaciones y nivel de gravedad de los pacientes que ingresan a la UCIC.

Un aumento en la cantidad de estados, para diferenciar de manera menos agregada los niveles de gravedad, como la definición de una etapa por periodos diarios o de medios días, pueden ser fácilmente incorporados sin alterar mayormente el modelo adoptado ni la complejidad del mismo.

Una extensión del modelo propuesto comprende métodos más complejos como aquellos basados en aprendizaje, como son los modelos Escondidos de Markov (Hidden Markov Models), pero cuya utilización en este primer trabajo de los autores está fuera del propósito de la presente investigación.

\section{AGRADECIMIENTOS}

Los autores desean agradecer muy sinceramente al Editor de la revista y a cada uno de los árbitros anónimos que participaron en su revisión, por sus valiosas observaciones y sugerencias que contribuyeron a mejorar la versión inicial de este artículo.

Los autores agradecen igualmente el apoyo de la Dirección General de Investigación y Postgrado de la Universidad Técnica Federico Santa María, a través del financiamiento otorgado al Proyecto de Investigación USM 28.04.21.

\section{REFERENCIAS}

[1] M.W. Carter. C.C. Price. "Operations Research: A practical introduction”. CRC Press. USA. 2001.

[2] D.J. White. "Further real applications of Markov decision processes". Interfaces, Vol. 18. $\mathrm{N}^{\mathrm{o}} 5$, pp. 55-61. 1988.

[3] F.A. Sonnenberg, J.R. Beck. "Markov models in medical decision making". Medical Decision Making, Vol. 13, pp. 322-338. 1993.

[4] N. Aikawa, S. Fujushima, M. Kobayashi, S. Matsuoka, T. Abiru. "Cost-minimization analysis of sivelestat for acute lung injury associated with systemic inflammatory response syndrome". Pharmacoeconomics. Vol. 23, $\mathrm{N}^{\mathrm{o}} 2$, pp. 169-181. 2005.

[5] R. Bauerle, A. Rucker, T.C. Schmandra, K. Holzer, A. Encke, E. Hanisch. "Markov cohort simulation study reveals evidence for sex-based risk difference in intensive care unit patients". American Journal of Surgery. Vol. 179, No 3, pp. 207-211. 2000.

[6] D. Collart, A. Haurie. "On the control of care supply and demand in a urology department". European Journal of Operational Research. Vol. 4; $\mathrm{N}^{\circ} 3$, pp. 160-172. 1980.

[7] R. Launois, M. Giroud, A.C. Mégnigbêto, K. Le Lay, G. Présenté, M.H. Mahagne, I. Durand, A.F. Gaudin. "Estimating the cost-effectiveness of stroke units in France compared with conventional care”. Stroke. Vol. 35, pp. 770-775. 2004.

[8] S.B. Patten. "Markov models of major depression for linking psychiatric epidemiology to clinical practice". Clinical Practice and Epidemiology in Mental Health. Vol. 1, No 2. 27 de abril de 2005. 1 de diciembre de 2005. http://www.cpementalhealth. com/content $/ \mathrm{I} / \mathrm{I} / 2$. 
[9] A. Shmueli, C.L. Sprung, E.H. Kaplan. "Optimizing admissions to an intensive care unit". Health Care Management Science. Vol. 6, pp. 131136. 2003.

[10] W.A. Knaus, E.A. Draper, D.P. Wagner, J.E. Zimmerman, M. Bergner, P.G. Bastos, C.A Sirio, D.J. Murphy, T. Lotring, A. Damiano et al. "The APACHE III prognostic system. Risk prediction of hospital mortality for critically ill hospitalized adults". Chest. Vol. 100, No 6, pp. 1619-1636. 1991.

[11] J.R. Le Gall, S. Lameshow, F. Saulnier. "A new Simplified Acute Physiology Score (SAPS II) based on European/North American multicenter study". The Journal of the American Medical Association. Vol. 270, pp. 2957-2963. 1993.

[12] S. Lemeshow, D. Teres, J. Klar, J.S. Avrunin, S.H. Gehlbach, J. Ropoport. "Mortality Probability Models (MPM II) based on an international cohort of intensive care unit patients". The Journal of the American Medical Association. Vol. 270, pp. 2478-2486. 1993.

[13] V. Parsonnet, D. Dean, A.D. Bernstein. "A method of unbiform stratification of risk for evaluating the results of surgery in acquired adult heart disease". Circulation. Vol. 79. Suppl. I, pp. 3-12. 1989.

[14] R.B. Becker, J.E. Zimmermann, W.A. Knaus, D.P. Wagner, M.G. Seneff, E.A. Draper, T.L Higgins, F.G. Estafanous, F.D. Loop. The use of APACHE III to evaluate ICU length of stay, resource use, and mortality after coronary artery bypass surgery. Journal of Cardiovascular Surgery. Vol. 36, pp. 1-11. 1995.

[15] D.R. Lawrance, O. Valencia, E.E. J. Smith, A. Murday, T. Treasure. "Parsonnet score is a good predictor of the duration of intensive care unit stay following cardiac surgery". Heart. Vol. 83, pp. $429-432.2000$.

[16] D.O. Clark, K. Kroenke, C.M. Callahan, C.J. McDonald. "Validity and Utility of Patientreported Health Measures on Hospital Admission". Journal of Clinical Epidemiology. Vol. 52, pp. 65-71. 1999

[17] P.L. Graham, D.A. Cook. "Prediction of Risk of Death Using 30-Day Outcome. A Practical End Point for Quality Auditing in Intensive Care". Chest. Vol. 125, pp. 1458-1466. 2004.
[18] K.H. Lee, G.D. Martich, A.J. Boujoukos, R.J. Keenan, and B.P. Griffith. "Predicting ICU length of stay following single lung transplantation". Chest. Vol. 110, pp. 1014-1017. 1996.

[19] J.V. Tu, S.B. Jaglal, C.D. Naylor. "Multicenter validation of a risk index for mortality, intensive care unit stay and overall hospital length of stay after cardiac surgery". Circulation. Vol. 91, pp. 677-684. 1995.

[20] G. Clermont, D.C. Angus, S.M. DiRusso, M. Griffin, W.T. Linde-Zwirble. "Predicting hospital mortality for patients in the intensive care unit: A comparison of artificial neural networks with logistic regression models". Critical Care Medicine. Vol. 29, pp. 291-296. 2001.

[21] M. Chacón, V. Rocco, E. Morgado, E. Sáez, S. Pliscoff. "Identificación de los determinantes de la estadía en unidades de cuidado intensivo usando redes neuronales artificiales". Revista Médica de Chile. Vol. 130, pp. 71-78. 2002.

[22] R. Scorpio, S. Walczak. "Predicting intensive care unit length of stay based on data upon arrival in the emergency room using a neural network. Critical Care Medicine. Vol. 26, Supplement, pp. 68A. 1998.

[23] A.S. Kapadia, S.E. Vineberg, C.D. Rossi. "Predicting course of treatment in a rehabilitation hospital: a markovian model". Computers \& Operations Research, Vol. 12, No 5, pp. 459-469. 1985.

[24] A.S. Kapadia, W. Chan, R. Sachdeva et al. "Predicting duration of stay in a pediatric intensive care unit: a markovian approach". European Journal of Operational Research. Vol. 124, pp. 353-359. 2000.

[25] D.P. Bertsekas, J.N. Tsitsiklis. "Introduction to Probability”. Athena Scientific. USA. 2002.

[26] S. Ross. "Introduction to Probability Models. Academic Press. USA. 2000.

[27] P. Peña. "Cadena de Markov para modelar la permanencia de pacientes en una unidad de cuidados intensivos cardiológicos". Memoria para optar al título de Ingeniero Comercial. Universidad Técnica Federico Santa María. 2002. 